\title{
The Aastrom experience
}

\author{
Ronnda L Bartel*, Caryn Cramer, Kelly Ledford, Amy Longcore, Christopher Parrish, Theresa Stern, Sharon Watling \\ and Frank Zeigler
}

\begin{abstract}
Aastrom Biosciences has developed a proprietary cellprocessing technology that enables the manufacture of ixmyelocel-T, a patient-specific multicellular therapy expanded from a small sample of a patient's own bone marrow. Ixmyelocel-T is produced under current good manufacturing practices (cGMP) in a fully closed, automated system that expands mesenchymal stem cells (MSCs) and macrophages. While the cell types in ixmyelocel-T are the same as those found in the bone marrow, the numbers of MSCs and alternative macrophages are greater in ixmyelocel-T. We propose that the mixture of expanded MSCs and alternatively activated macrophages promote long-term tissue repair of ischemic tissue. The multiple cell types in ixmyelocel-T have a range of biological activities that are likely to contribute to a complex mechanism of action. Clinical trial data collected to date support the potential for ixmyelocel-T as an efficacious and safe treatment for ischemic cardiovascular indications, including critical limb ischemia (CLI) and a severe form of heart failure, dilated cardiomyopathy (DCM). The CLI clinical program has completed phase 2 and has reached concurrence with the Food and Drug Administration (FDA) on a phase 3 study (REVIVE) through the Special Protocol Assessment (SPA) process. The phase 3 study began screening patients in February 2012. The DCM clinical program will initiate phase 2b in 2012.
\end{abstract}

\section{Introduction}

Aastrom Biosciences, founded in 1989, has developed a proprietary cell-processing technology that enables the manufacture of ixmyelocel-T, a patient-specific multicellular therapy expanded from a patient's own bone marrow. Aastrom has developed a bioreactor specifically

*Correspondence: rbartel@aastrom.com

Aastrom Biosciences, Inc., Domino's Farms, Lobby K, 24 Frank Lloyd Wright Drive, Ann Arbor, MI 48105, USA designed for the ex vivo expansion of autologous marrowderived stem and progenitor cells that mimics the in vivo environment of bone marrow tissue. Two key cell types are expanded in this $12 \pm 1$-day culture process: stromal cells, including mesenchymal stem cells (MSCs), and alternatively activated macrophages. Aastrom has advanced ixmyelocel-T into late-stage clinical development in critical limb ischemia (CLI), including the completion of a phase 2 trial and concurrence with the US Food and Drug Administration (FDA) on a phase 3 study through the Special Protocol Assessment (SPA) process. In addition, the phase $2 \mathrm{~b}$ clinical program in patients with dilated cardiomyopathy (DCM) will initiate in 2012. Other areas of research are ongoing.

Adult stems cells can be found in the central nervous system, skeletal muscles, pancreas, liver, adipose tissue, and the bone marrow and blood. Stem cells found in the bone marrow have been studied for almost 50 years [1]. Two primary types of stem cells are found in the bone marrow: hematopoietic stem cells (HSCs), and stromal cells (including MSCs, multipotent stromal cells, and endothelial progenitors).

HSCs are responsible for forming all the types of blood cells in the body. Approved treatment uses for stem cells are primarily for HSCs collected from the bone marrow or the peripheral blood for the treatment of specific types of cancers (leukemia, lymphoma, and myeloma). Stromal cells are a mixed population of support cells that generate the regulatory niches that support blood cell formation from HSCs [2]. Recently, progenitor cells with the capacity to differentiate into vascular endothelial cells have been identified in bone marrow and peripheral blood, but this population appears to be restricted to the endothelial lineage and would therefore be a committed precursor. While a committed endothelial progenitor is not a 'stem cell' per se, it is a cell type that can be therapeutically effective without the need to strictly maintain multipotentiality, in theory providing a source of new blood vessels [3].

Aastrom scientists have focused their research on the expansion of adult cells taken from the bone marrow of an individual patient. The expanded cell product, ixmyelocel-T, is then injected into ischemic tissue in the same patient. 


\section{Manufacturing}

The challenge in the collection of autologous stem cells for treatment lies in the rarity of adult stem cells. For example, only an estimated 1 in 10,000 to 15,000 cells in bone marrow is an HSC [4]. MSCs are approximately ten-fold less abundant in bone marrow than HSCs [5]. Taking large (up to $1,000 \mathrm{ml}$ in some cases [6]) volumes of bone marrow from a patient is a painful process that requires general anesthesia and hospitalization. Therefore, taking a small sample of bone marrow from a patient and expanding the cell populations ex vivo is an obvious benefit to the patient. Ixmyelocel- $T$ is manufactured from a small sample (approximately $60 \mathrm{ml}$ ) of autologous bone marrow aseptically withdrawn from the posterior iliac crest of a patient under local anesthesia and conscious sedation during a 20-minute outpatient procedure. The bone marrow aspirate sample is shipped overnight to Aastrom's manufacturing facility in an insulated shipping container supplied by Aastrom that is qualified to maintain the aspirate at ambient temperature. Aastrom is in a unique position in the field of cell therapy, having developed a product under current good manufacturing practices (cGMP) in a fully closed, automated system for the expansion of stem and progenitor cells [7].

Ixmyelocel- $T$ is produced by placing collected bone marrow aspirate cells into a bioreactor system under controlled conditions, and harvesting the cells after a specified amount of time. The ixmyelocel-T manufacturing process is initiated by using an automated, closed system (SEPAX Cell Separation System manufactured by Biosafe, Houston, TX, USA) to perform a Ficoll-based density gradient centrifugation process to deplete red blood cells and purify bone marrow mononuclear cells (BMMNCs). The purified BMMNCs are collected and transferred into a single-use, sterile, disposable cell cassette that is a component of Aastrom's proprietary, automated, closed cell processing system. The system uniformly distributes the cells over the culture surface and then controls the culture conditions, including temperature, culture medium exchange, and gas exchange.

After $12 \pm 1$ days of culture, cells are washed and harvested from the cassette by a multistep, automated process. The cells are then concentrated by centrifugation to a final volume suitable for patient administration. Ixmyelocel- $\mathrm{T}$ is shipped overnight to the treatment site using a qualified gel-packed shipping container that maintains the product at hypothermic temperature; in this container the product has a 72-hour shelf life. Components of the manufacturing system are pictured in Figure 1 [8].

\section{Evidence of an effect: characterization of ixmyelocel-T and preclinical research}

Because of the mixed cell composition of ixmyelocel-T, a wide range of biological activities relevant to the repair and regeneration of ischemic tissue are expected. These biological activities include tissue remodeling and immunomodulation, which may promote and support angiogenesis in a synergistic manner $[9,10]$. The role of MSCs in tissue repair is at least partially due to their consistent trophic effects mediated by the wide range of growth factors and cytokines they produce [11]. The following presents an overview of the characteristics of ixmyelocel-T, and the multiple mechanisms by which ixmyelocel-T may impact the repair of damaged ischemic tissue.

\section{Characterization of the cell populations}

Ixmyelocel- $T$ is composed of a mixture of cell types that include those expected to be found in the BMMNC population. These include myeloid cells (that is, granulocytes, monocytes, and mixed myeloid progenitors) and lymphoid cells (that is, T cells, B cells, and mixed lymphoid progenitors) that express CD45 on the cell surface and $\mathrm{CD} 90^{+} \mathrm{MSCs} /$ stromal cells, and CD $45^{+} \mathrm{CD} 14^{+}$ autofluorescent ${ }^{+}\left(\mathrm{CD}_{14}{ }^{+}\right.$Auto $\left.^{+}\right)$macrophages. While the cell types are similar to those found in the BMMNC population, the numbers of $\mathrm{CD}^{+} 0^{+}$and $\mathrm{CD} 14^{+} \mathrm{Auto}^{+}$cells are significantly greater in ixmyelocel- $\mathrm{T}$ due to expansion during the manufacturing process.

Figure 2a-d shows a graphical representation of the changes in the cell types during the culture process, including the fold-change in cell phenotypes. A summary of the cell phenotypes present in ixmyelocel- $\mathrm{T}$ is presented in Figure 3.

The prevailing scientific view is that a mixture of regenerative cell types like MSCs and alternatively activated macrophages $\left(\mathrm{CD} 0^{+}\right.$and $\mathrm{CD} 14^{+} \mathrm{Auto}^{+}$, respectively), rather than a single cell type, are required to promote long-term tissue regeneration and repair $[9,10]$. Though not within the scope of this review, a significant body of scientific research supports the role of bone marrowderived MSCs and myeloid cells in the restoration of blood flow to chronic ischemic tissues. Bone marrow maintains a small reservoir of these two cell types that the Aastrom process significantly expands while maintaining many of the mononuclear cells from the original bone marrow sample.

Not unexpectedly, these diverse cell types display a wide range of biological activities relevant to the repair and regeneration of ischemic tissue, including tissue remodeling and immunomodulation and the promotion of angiogenesis. We propose that these activities are the most likely responsible for the complex and multiple mechanisms of action in this multicellular therapy.

\section{Tissue remodeling}

Ixmyelocel-T contains large numbers of alternatively activated macrophages and MSCs, both of which are cell 


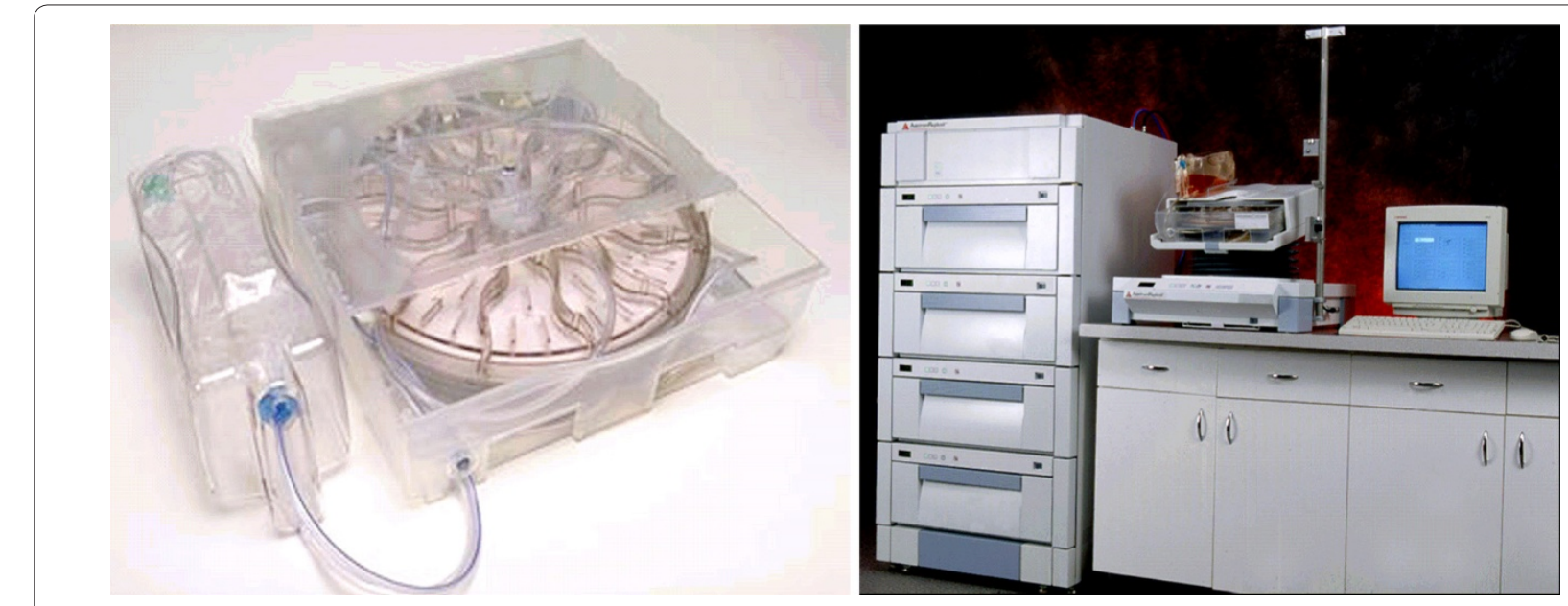

Figure 1. Aastrom manufacturing platform. Left: single-use cell cassette. Right: highly automated instrument platform.

(a)

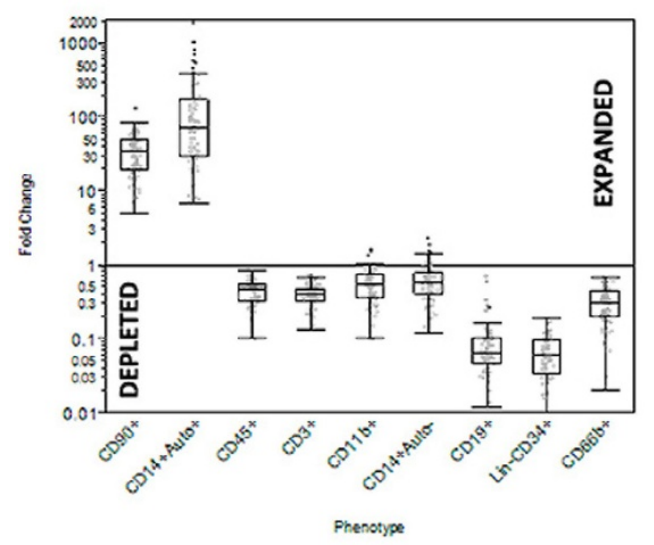

(c)

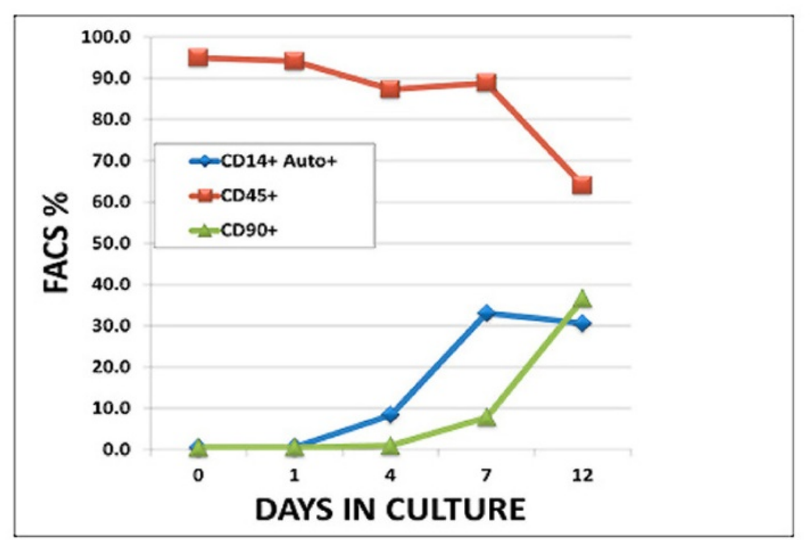

(b)

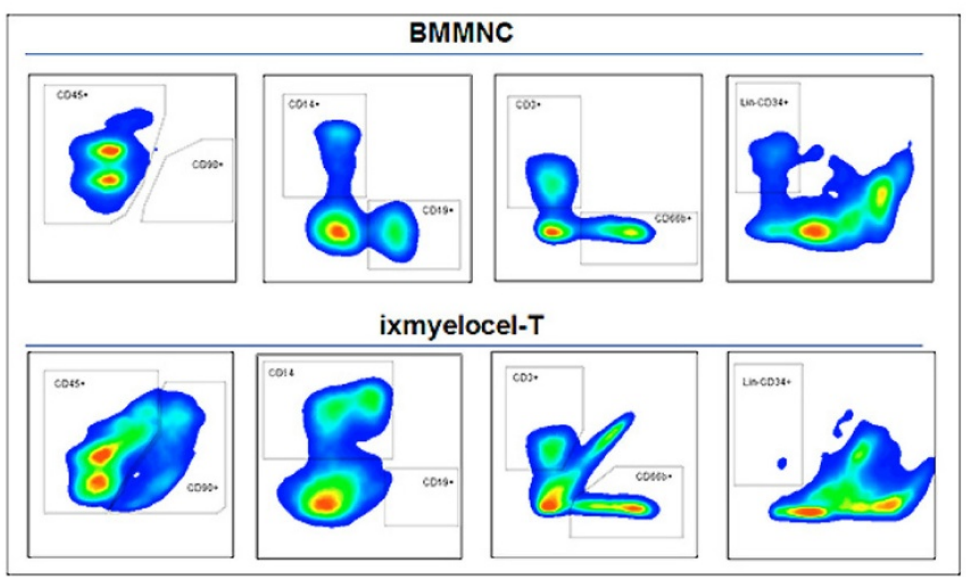

(d)

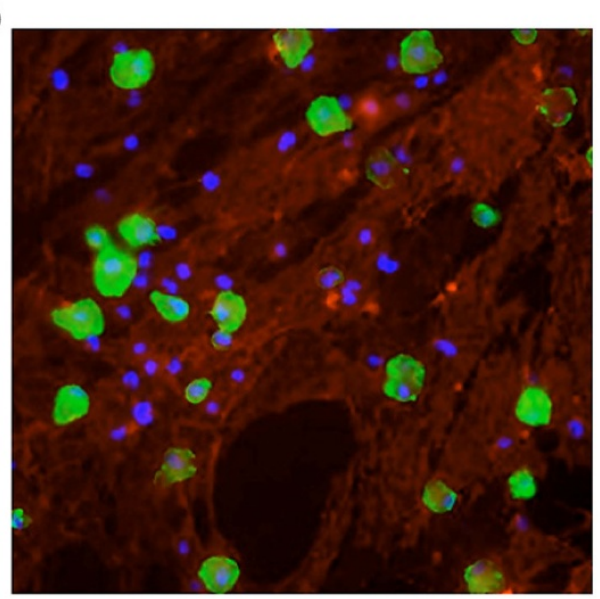

Figure 2. Changes in cell types during the culture process for ixmyelocel-T. (a) Fold change in cell phenotypes of the expanded and depleted cells in ixmyelocel-T. (b) Comparison of bone marrow mononuclear cells (BMMNCs) and ixmyelocel-T from a matched donor by flow cytometry. (c) Kinetics of expansion of $\mathrm{CD} 90^{+}$mesenchymal stem cells and CD $14^{+}$Auto ${ }^{+}$activated macrophages and overall loss of CD45 cells over time during ixmyelocel-T. (d) Immunofluorescence image of CD90 (red), CD14 (green), and nuclei (blue) at 600x. FACS, fluorescence-activated cell sorting. 


\begin{tabular}{|l|l|l|l|l|l|}
\hline \multicolumn{2}{|c|}{ Cell Type(s) } \\
\hline \%
\end{tabular}

Figure 3. Ixmyelocel-T cell phenotypes. NK, natural killer.

types that may promote remodeling of the extracellular matrix (ECM) in ischemic tissue. The ECM regulates many aspects of cell behavior and within a single tissue, it is constantly being remodeled as cells build and reshape their environment. Remodeling rates are particularly high during periods of infection and wound repair [12]. Using proteomic techniques, ixmyelocel- $\mathrm{T}$ has been shown to secrete a variety of ECM proteins and matrix metalloproteins (MMPs) that remodel the ECM [13]. Additionally, the MSCs in ixmyelocel-T have been shown to maintain a significant osteoblastic potential and, when combined with a bone inductive matrix, efficiently regenerate bone in a mouse calvarial defect model. The MSCs from ixmyelocel- $T$ have also been shown to engraft and participate in new bone formation based on localization and expression of a bone-specific ECM gene linked to a fluorescent reporter gene [14].

The $\mathrm{CD} 14^{+}$macrophages in ixmyelocel-T are actively phagocytic, and this property could provide for clearance of necrotic tissue and apoptotic cells in diseased or damaged tissues. Figure 4 shows that the CD14 cells actively internalize acetylated low-density lipoprotein as measured by flow cytometry.

\section{Immunomodulation}

Successful repair and regeneration of ischemic tissue requires the removal of damaged or necrotic cells and tissue, as well as the resolution of pathological inflammation. Ixmyelocel-T may promote vascular recovery by

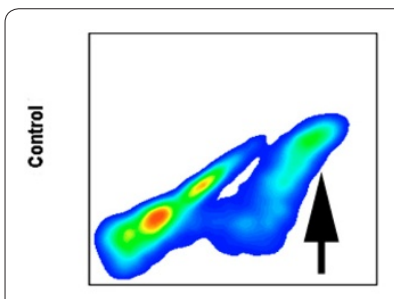

AcLDL AF488

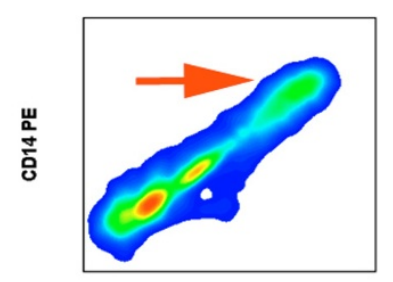

AcLDL AF488
Figure 4. Fluorescence-activated cell sorting analysis for acetylated low-density lipoprotein (AcLDL) uptake shows ixmyelocel-T macrophages are active phagocytes. The phagocytic cells that are Ac-LDL+ (black arrow) are all CD14+ (red arrow).

exerting protective effects on the endothelium through a population of alternatively activated macrophages that regulate inflammation and tissue remodeling. Activated macrophages can be proinflammatory (classical or M1 type macrophages, associated with $\mathrm{T}$ helper 1 lymphocytes (Th1)) or can be alternatively activated and antiinflammatory (alternative or M2 type macrophages, associated with Th2). Macrophages that are M2 have been associated with the resolution of inflammation and progression of tissue repair and healing after ischemic damage or infection. Alternatively activated macrophages in ixmyelocel-T express scavenger receptors CD163 and CD206 (Figure 5), consistent with an alternative activation phenotype (M2). 


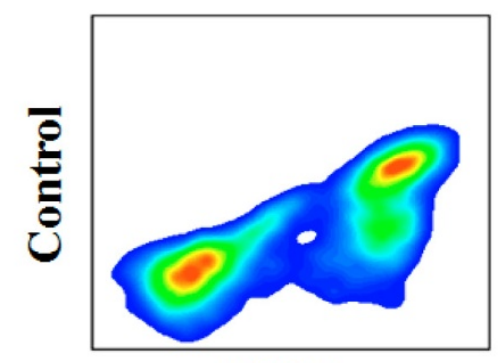

CD14

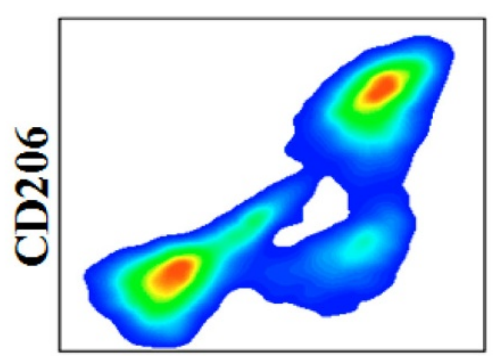

CD14

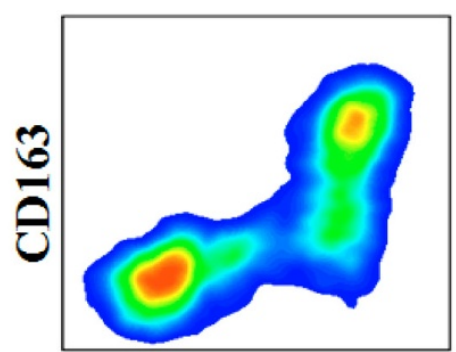

CD14

Figure 5. Ixmyelocel-T CD14+ cells co-express CD206 and CD163.

Analysis of the cytokine secretion profile of ixmyelocel$\mathrm{T}$ has been shown to be consistent with the Th2/M2 phenotype. The pro-inflammatory cytokines IL- $1 \alpha$ and IL-1 $\beta$ are barely detected (approximately $<10 \mathrm{pg} / \mathrm{ml}$ ), while anti-inflammatory cytokines IL-10 and IL-1 receptor antagonist (IL-1ra) are actively secreted by ixmyelocel$\mathrm{T}$, primarily by the $\mathrm{CD} 14^{+} \mathrm{Auto}^{+}$cells (approximately 5,000 to $10,000 \mathrm{pg} / \mathrm{ml}$ ). The lack of Th1/M1 proinflammatory cytokines (IL- $1 \alpha$ and IL-1 $\beta$ ), and a highlevel secretion of Th2/M2 anti-inflammatory cytokines (IL-10 and IL-1ra) demonstrate that ixmyelocel-T is likely promoting resolution of inflammation rather than further contributing to pathological inflammation.

Multiplex measurement of secreted cytokines from ixmyelocel-T product has been shown in sorted cell populations. Ixmyelocel-T secretes a distinct and characteristic array of cytokines, chemokines and growth factors, including biologically significant levels of angiogenic (vascular endothelial growth factor, angiopoietin 1, angiopoietin 2, hepatocyte growth factor, IL-8) and antiinflammatory or immune regulatory factors (adiponectin, IL-1ra, IL-6, IL-10, IP-10, monocyte chemotactic protein-1 and transforming growth factor- $\beta$ ) together with low or undetectable levels of pivotal pro-inflammatory cytokines (such as IL-1 $\alpha$, IL-1 $\beta$, IL-15, IL-17, tumor necrosis factor- $\alpha$ and most notably lacking interferon and IL-12 as quantified by ELISA and Luminex-based assays). A summary of the multiplex cytokine analysis of BMMNCs compared to $\mathrm{CD} 0^{+}$and $\mathrm{CD} 14^{+} \mathrm{Auto}^{+}$from ixmyelocel-T is shown in Figure 6.

Aastrom is continuing to evaluate the properties of ixmyelocel-T through in vitro cell biology as well as testing in relevant animal models. A summary of clinical trial data collected for the two ongoing cardiovascular programs (DCM and CLI) are described below.

\section{Evidence of an effect: clinical research Dilated cardiomyopathy program}

DCM is a form of heart failure; it is a progressive disease, the third most common cause of heart failure and the most frequent cause of heart transplantation [15]. DCMs are associated with both systolic abnormalities (difficulty of the left ventricle to empty or eject blood from its chamber) and diastolic abnormalities (increased resistance to filling of one or both ventricles). Heart enlargement and poor function generally lead to progressive heart failure with further decline in the ability of the heart to contract and pump blood around the body efficiently.

Ixmyelocel-T received orphan product designation for the treatment of DCM in 2007. Aastrom has conducted an exploratory phase 2 a program in patients with heart failure due to DCM using intramyocardial surgical and transendocardial catheter delivery of ixmyelocel-T. To date, two phase $2 \mathrm{a}$ open-label studies using the two different delivery methods (Study ABI-55-0712-1 (IMPACT-DCM) surgical delivery, and Study ABI-55-0811-1 (CATHETER-DCM) catheter delivery) have been conducted. Both studies were open-label, multicenter, randomized (ixmyelocel-T or standard of care) studies in patients with a diagnosis of ischemic DCM (IDCM) or nonischemic DCM (NIDCM). The numbers of patients in each study are presented in Figure 7.

The CATHETER-DCM study is ongoing. The results of the 12-month data for the surgical IMPACT-DCM study were presented via poster at the Heart Failure Society of America meeting in September 2011 [16]. Since the IMPACT-DCM trial was an open-label exploratory study, the efficacy conclusions that can be made from evaluation of the data are limited. As shown in Table 1, however, there were consistent trends showing the potential benefit of treatment with ixmyelocel-T. In the ischemic population, major adverse cardiac events were experienced by a lower percentage of ixmyelocel-T-treated patients compared to control patients. This difference in major adverse cardiac events between ixmyelocel-Ttreated and control ischemic patients was further increased when the surgical day (day 0) was eliminated from the analysis (days 1 to 365). The majority of 


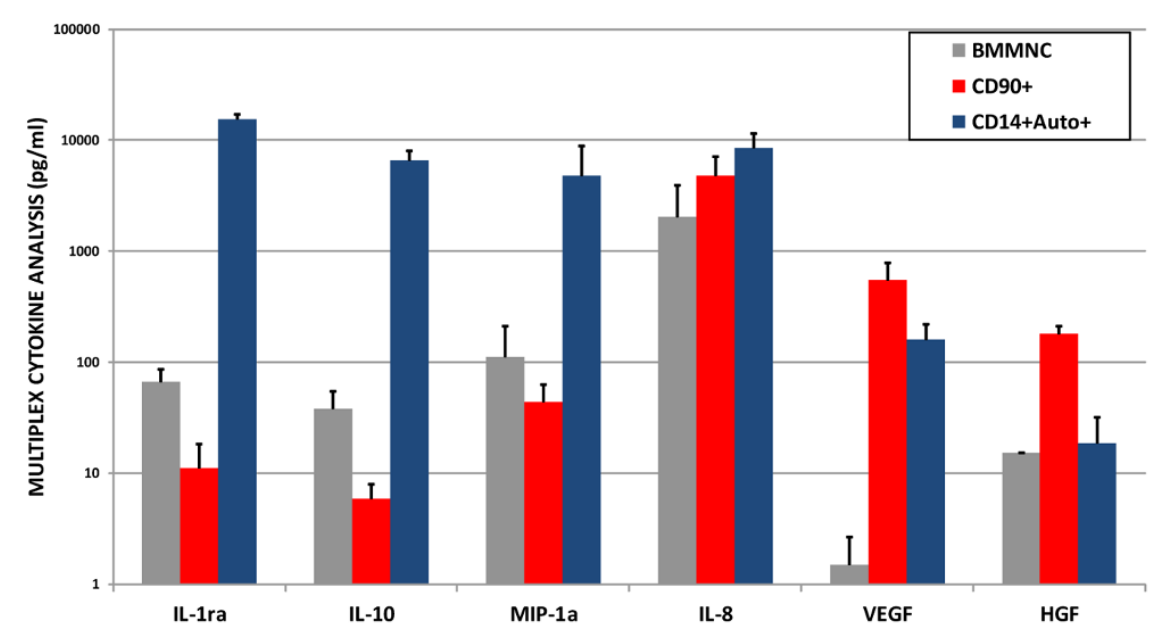

Figure 6. Summary of the multiplex cytokine analysis of bone marrow mononuclear cells (BMMNCs) compared to ixmyelocel-T CD90 ${ }^{+}$ and $\mathrm{CD} 14^{+}$Auto populations. Cells were cultured for approximately 24 hours in multiwell plates and cytokine levels were measured in comparison to blank medium negative controls. $\mathrm{CD} 90^{+}$and $\mathrm{CD} 14^{+} \mathrm{AutO} \mathrm{O}^{+}$populations were sorted using fluorescence-activated cell sorting to high purity prior to culture. Non-paired data from two or more independent donors assayed in replicate are expressed as the mean cytokine concentration \pm standard error in pg/ml. IL-1 ra, IL-1 receptor antagonist; HGF, hepatocyte growth factor; MIP, macrophage inflammatory protein; VEGF, vascular endothelial growth factor.

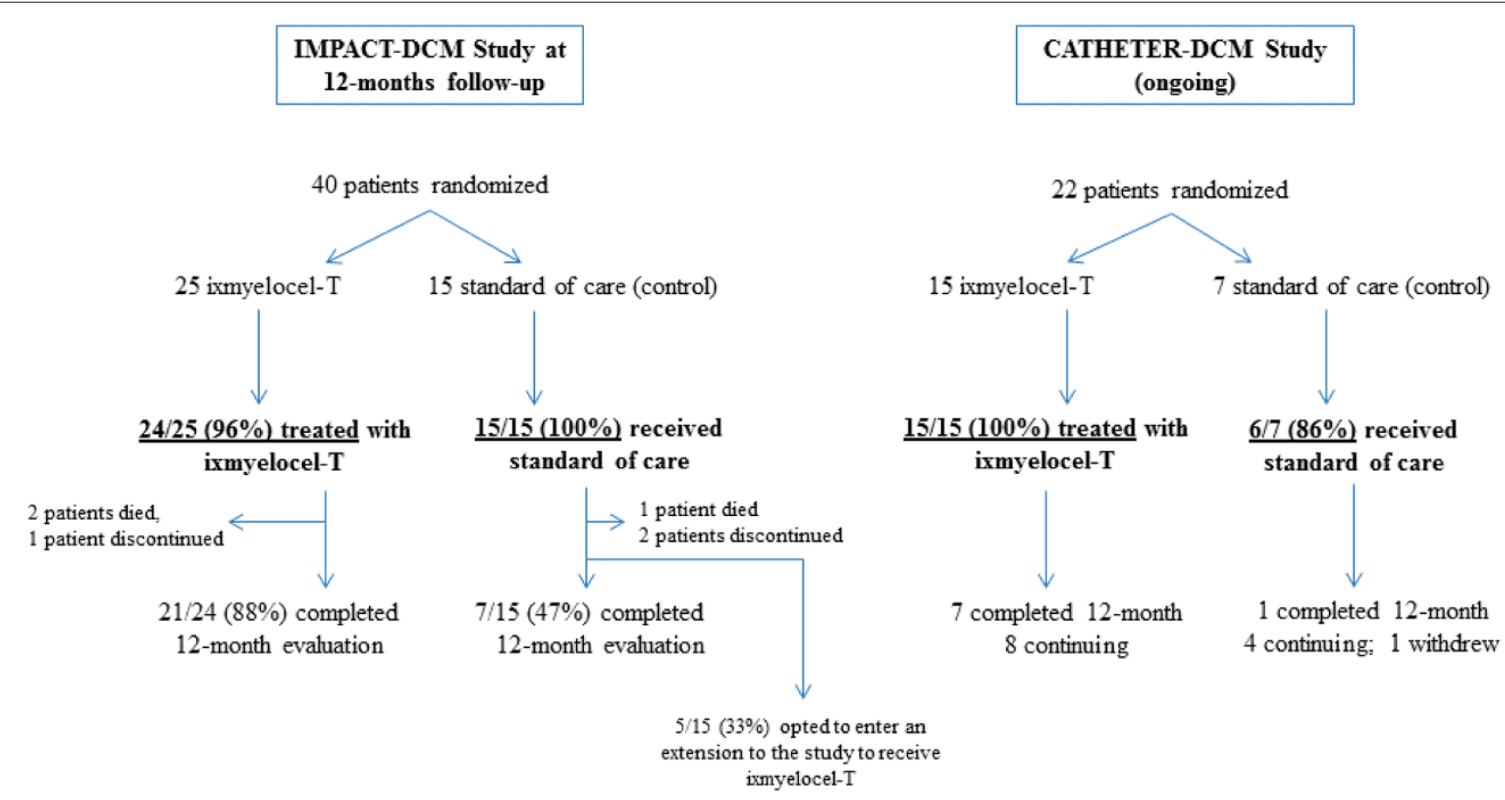

Figure 7. Flow diagram of the IMPACT-DCM and CATHETER-DCM studies. DCM, dilated cardiomyopathy.

ixmyelocel-T-treated patients (both IDCM and NIDCM) had improvement in New York Heart Association class over the 12 months following treatment. Eight patients (five IDCM and three NIDCM) receiving ixmyelocel-T had an improvement of two classes (from class III to class I) from screening to 12 months. There was also a trend toward improved function, with a higher percentage of ixmyelocel-T-treated IDCM patients showing an increased 6-minute walk performance compared to the IDCM control patients. Left ventricular structural indices showed a trend toward improvement in septal thickness in the IDCM patients.

\section{Critical limb ischemia program}

CLI is the most severe form of peripheral arterial disease that results from markedly reduced blood flow to the legs, feet, and hands and is usually caused by atherosclerosis. An estimated 3 to $5 \%$ of adults aged 40 years 
Table 1. Summary of efficacy - all treated patients (IMPACT-DCM)

\begin{tabular}{|c|c|c|c|c|c|}
\hline & & \multicolumn{2}{|c|}{ IDCM } & \multicolumn{2}{|c|}{ NIDCM } \\
\hline & & Ixmyelocel-T & Control & Ixmyelocel-T & Control \\
\hline \multicolumn{6}{|l|}{ Percent of patients with MACE adverse events } \\
\hline Enrolled patients & & 12 & 7 & 12 & 8 \\
\hline All MACE events ( $(\%))$ & & $6(50.0)$ & $5(71.4)$ & $5(41.7)$ & $2(25.0)$ \\
\hline Total MACE events & & 8 & 8 & 8 & 3 \\
\hline Event on day 0/injection (n (\%)) & & $2(16.7)$ & 0 & $1(8.3)$ & 0 \\
\hline Total MACE events day 0/injection & & 2 & 0 & 1 & 0 \\
\hline Event on days 1 to $365(n(\%))$ & & $3(25.0)$ & $4(57.1)$ & $4(33.3)$ & $1(12.5)$ \\
\hline Total MACE events days 1 to 365 & & 5 & 7 & 7 & 1 \\
\hline Event on day $366+(n(\%))$ & & $1(8.3)$ & $1(14.3)$ & 0 & $1(12.5)$ \\
\hline Total MACE events day $366+$ & & 1 & 1 & 0 & 2 \\
\hline \multicolumn{6}{|c|}{ Number of patients with improvements from baseline in efficacy/total patients in group } \\
\hline & 12 months & $9 / 10$ & $1 / 3$ & $7 / 9$ & $2 / 3$ \\
\hline Increase in six minute walk test & 12 months & $6 / 10$ & $0 / 3$ & $8 / 9$ & $3 / 3$ \\
\hline Increase in echocardiogram ejection fraction & 12 months & $3 / 7$ & $0 / 2$ & $4 / 7$ & $1 / 2$ \\
\hline
\end{tabular}

IDCM, ischemic dilated cardiomyopathy; MACE, major adverse cardiac event; NIDCM, non-ischemic dilated cardiomyopathy; NYHA, New York Heart Association.

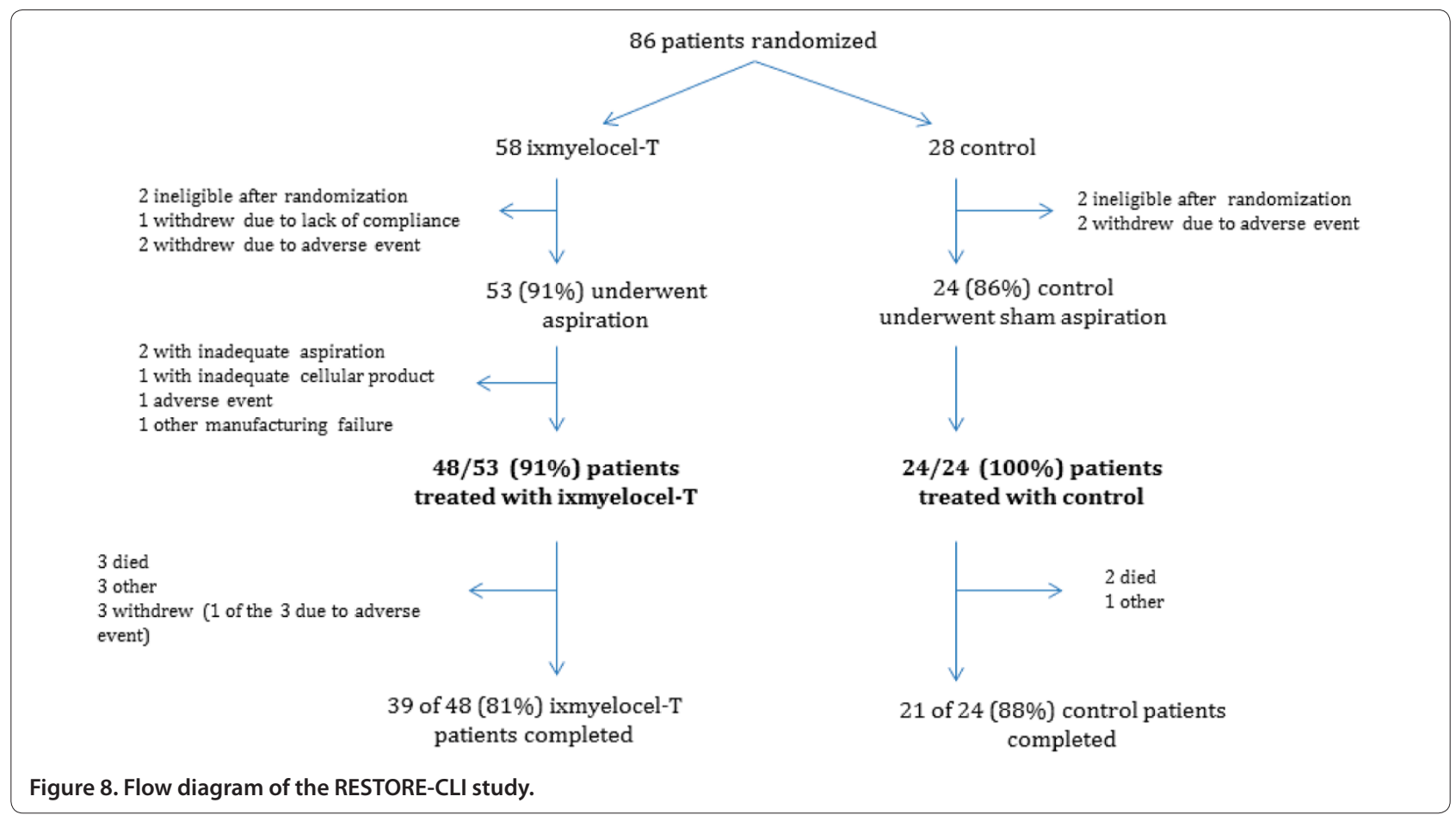

and older have peripheral arterial disease in the United States [17]. It is estimated that $5 \%$ to $10 \%$ of peripheral arterial disease patients over 50 years of age will develop CLI within 5 years [18]. Many patients with CLI have multiple co-morbidities, which may often prevent them from having open bypass or endovascular surgical procedures. It is estimated that up to $40 \%$ of CLI patients are not candidates for surgery [19]. Major amputation is necessary when there is overwhelming infection that threatens the patient's life, when rest pain cannot be controlled, or when there is extensive skin and tissue loss. 


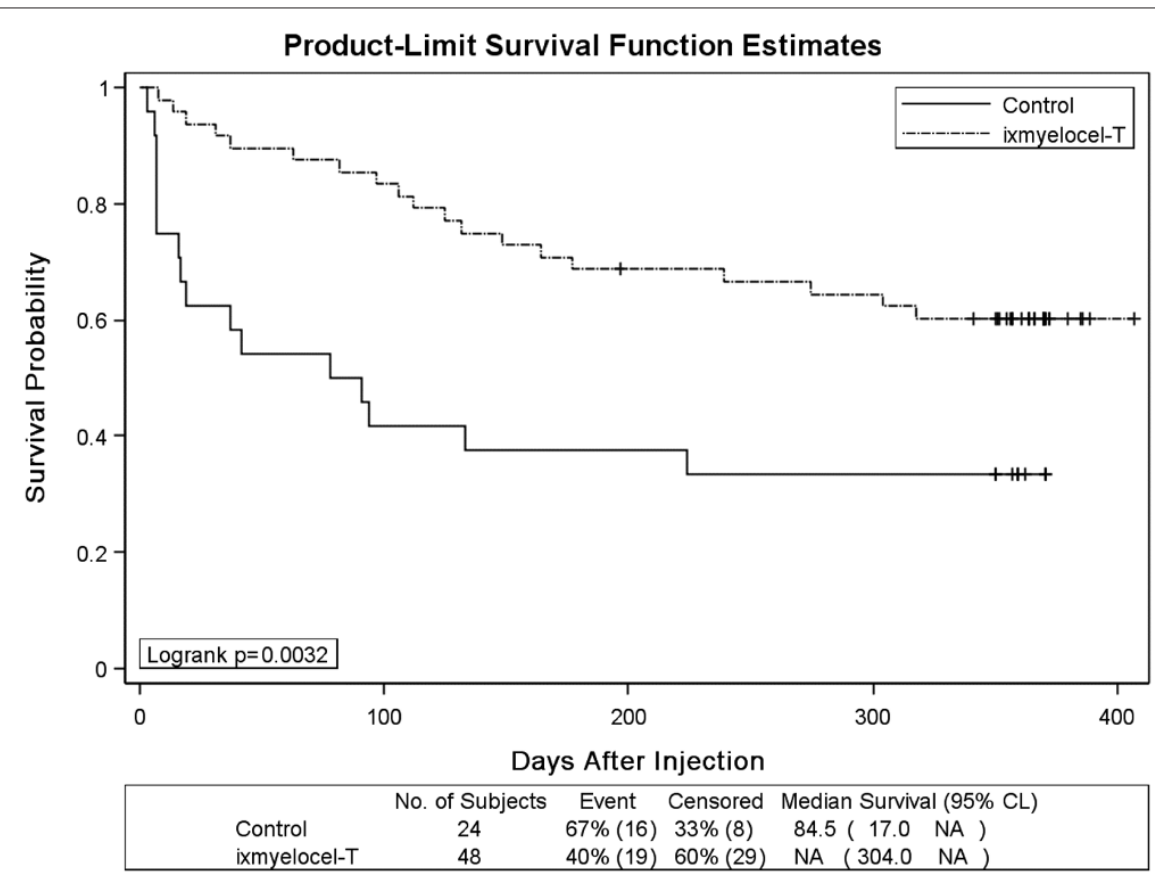

Figure 9. Kaplan-Meier curve: time to treatment failure (RESTORE-CLI). Kaplan-Meier survival plot of time to treatment failure (major amputation of injected leg, all-cause mortality, doubling of total wound surface area from baseline, de novo gangrene) for all patients injected. Censored observations are indicated by plus symbols. CL, confidence limit; NA, not available. Reprinted with permission of the author [21].

A phase $2 \mathrm{~b}$ clinical study has been successfully completed in the CLI program with positive results presented at the American Heart Association Scientific Sessions 2011 in November 2011 [20,21]. A pivotal phase 3 clinical study under a SPA approved by the FDA began screening patients in early 2012. The phase 2 study (RESTORECLI) was a randomized, double-blind, placebo-controlled study in patients with no options for revascularization. Safety data were evaluated from 77 aspirated patients (53 ixmyelocel-T and 24 control); efficacy data were evaluated in 72 treated patients (48 ixmyelocel- $\mathrm{T}$ and 24 control) (Figure 8).

As expected with an autologous cell product, the safety profile showed that the ixmyelocel-T was well tolerated, with a similar adverse event and serious adverse event profile in the ixmyelocel- $T$ group relative to the control group. RESTORE-CLI was not powered to show statistical significance for efficacy endpoints; despite that limitation, however, there was a statistically significant difference in the time to first occurrence of treatment failure. The treatment failure composite, which consisted of major amputation of the index leg, all-cause mortality, doubling of wound total surface area from baseline and de novo gangrene, is a phase 2 surrogate for the phase 3 amputation-free survival (major amputation of the index leg, all-cause mortality) endpoint. Time to first occurrence of treatment failure is the earliest day at which any of the treatment failure events occurred. There was a $62 \%$
Table 2. Contribution to treatment failure composite endpoint in treated patients (Ixmyelocel-T or control)

\begin{tabular}{lcc}
\hline Endpoint: $\boldsymbol{n}(\%)$ & $\begin{array}{c}\text { Ixmyelocel-T } \\
\mathbf{N}=\mathbf{4 8}\end{array}$ & $\begin{array}{c}\text { Control } \\
\mathbf{N}=\mathbf{2 4}\end{array}$ \\
\hline Major amputation & 6 & 4 \\
All-cause mortality & 2 & 1 \\
Doubling in total wound surface area & 5 & 7 \\
De novo gangrene & 6 & 4 \\
Total & $19(39.6 \%)^{\mathrm{b}}$ & $16(66.7 \%)^{\mathrm{b}}$
\end{tabular}

apatient must have come into the study with a wound to be eligible to contribute to this event. ${ }^{b} P=0.0451$, Fisher's exact test.

risk reduction in treatment failure over the 12-month follow-up in the ixmyelocel-T group compared to the control group (hazard ratio $0.38,95 \%$ confidence interval $=$ 0.20 to 0.74; Figure 9). As shown in Table 2, the individual components of the treatment failure composite endpoint all trended in the same direction, favoring ixmyelocel-T treatment, with the exception of all-cause mortality that was the same in both treatment groups.

\section{Conclusions}

Ixmyelocel-T is a patient-specific multicellular therapy expanded from a patient's own bone marrow. The product is manufactured using a fully closed, highly automated bioreactor system specifically designed for the ex vivo expansion of autologous marrow-derived stem 
and progenitor cells that mimics the in vivo environment of bone marrow tissue. Characterization of ixmyelocel-T has shown a mixture of MSCs and alternatively activated macrophages that have a wide range of biological activities relevant to the repair and regeneration of ischemic tissue. This mixture of cell types has multiple mechanisms of action, including tissue remodeling and immunomodulatory functions that target the many underlying causes of severe, chronic cardiovascular diseases. Clinical trial data collected to date support the potential for ixmyelocel- $T$ as an efficacious and safe treatment for CLI and DCM; however, data from phase 3 clinical programs are needed for confirmation.

This article is part of a thematic series on Clinical applications of stem cells edited by Mahendra Rao. Other articles in the series can be found online at http://stemcellres.com/series/clinical

\section{Abbreviations}

BMMNC, bone marrow mononuclear cells; CLI, critical limb ischemia; DCM, dilated cardiomyopathy; ECM, extracellular matrix; ELISA, enzymelinked immunosorbent assay; FDA, Food and Drug Administration; HSC, hematopoietic stem cell; IDCM, ischemic dilated cardiomyopathy; IL, interleukin; IL-1 ra, IL-1 receptor antagonist; MSC, mesenchymal stem cell; NIDCM, non-ischemic dilated cardiomyopathy; SPA, Special Protocol Assessment; Th, Thelper

\section{Competing interests}

All authors are employees of Aastrom Biosciences. Ixmyelocel-T is protected by patents US 7,871,605 and EP246551 issued in 2011 "Mixed cell populations for tissue repair and separation technique for cell processing".

Published: 9 July 2012

\section{References}

1. Friedenstein AJ, Chailakhjan RK, Lalykina KS: The development of fibroblast colonies in monolayer cultures of guinea-pig bone marrow and spleen cells. Cell Tissue Kinetics 1970, 3:393-403.

2. Williams A, Hare JM: Mesenchymal stem cells: biology, pathophysiology, translational findings, and therapeutic implications for cardiac disease. Circ Res 2011, 109:923-940.

3. National Institutes of Health: The Adult Stem Cell (Stem Cell Information) [http://stemcells.nih.gov/info/scireport/chapter4]

4. Weissman LL: Stem cells: units of development, units of regeneration, and units in evolution. Cell 2000, 100:157-168.

5. Shi Y, Hu G, Su J, Li W, Chen Q, Shou P, Xu C, Chen X, Huang Y, Zhu Z, Huang X, Han X, Xie N, Ren G: Mesenchymal stem cells: a new strategy for immunosuppression and tissue repair. Cell Res 2010, 20:510-518.

6. Lannert H, Able T, Becker S, Sommer M, Braun M, Stadtherr P, Ho AD: Optimizing BM harvesting from normal adult donors. Bone Marrow Transplant 2008, 42:443-447.
7. Mandalam RK, Koller MR, Smith AK: Ex vivo hematopoietic expansion for bone marrow transplantation. In Ex Vivo Cell Therapy. Edited by Schindhelm K, Nordon R. San Diego: Academic Press; 1999:273-291.

8. Goltry K, Hampson B, Venturi N, Bartel R: Large-scale production of adult stem cells for clinical use. In Emerging Technology Platforms for Stem Cells. Edited by Lakshmipathy U, Chesnut JD, Thyagarajan B. John Wiley and Sons, Inc.; 2009:153-168.

9. van Weel V, van Tongeren RB, van Hinsbergh VW, van Bockel JH, Quax PH: Vascular growth in ischemic limbs: a review of mechanisms and possible therapeutic stimulation. Ann Vasc Surg 2008, 22:582-597.

10. Shireman PK: The chemokine system in arteriogenesis and hind limb ischemia. J Vasc Surg 2007, 45(Suppl A):A48-A56.

11. Caplan Al, Dennis JE: Mesenchymal stem cells as trophic mediators. J Cell Biochem 2006, 98:1076-1084.

12. Daley WP, Peters SB, Larsen M: Extracellular matrix dynamics in development and regenerative medicine. J Cell Sci 2007, 121:255-264.

13. Prahalad AK, Hock JM: Proteomic characteristics of ex vivo-enriched adult human bone marrow mononuclear cells in continuous perfusion cultures. J Proteome Res 2009, 8:2079-2089.

14. Yin D, Wang Z, Gao Q, Sundaresan R, Parrish C, Yang Q, Krebsbach PH, Lichtie AC, Rowe DW, Hock J, Liu P: Determination of the fate and contribution of ex vivo expanded human bone marrow stem and progenitor cells for bone formation by 2.3ColGFP. Mol Ther 2009, 17:1967-1978.

15. Goswami V: Dilated Cardiomyopathy (Medscape Reference: Drugs, Diseases and Procedures) [http://emedicine.medscape.com/article/152696-overview]

16. Patel A, Hamman B, Bruckner B, Lattouf O, Smedira N, Bartel R, Watling S: Safety and efficacy of ixmyelocel-T, an expanded patient-specific mixed cell product, in dilated cardiomyopathy (IMPACT-DCM). J Cardiac Failure 2011, 17(Suppl):S58.

17. Selvin E, Erlinger TP: Prevalence of and risk factors for peripheral arterial disease in the United States: results from the National Health and Nutrition Examination Survey, 1999-2000. Circulation 2004, 110:738-743.

18. Norgren L, Hiatt WR, Dormandy JA, Nehler MR, Harris KA, Fowkes FG; TASC II Working Group: Inter-society consensus for the management of peripheral arterial disease (TASC II). Eur J Vasc Endovasc Surg 2007, 33 Suppl:S1-S75.

19. Powell RJ, Simons M, Mendelsohn FO, Daniel G, Henry TD, Koga M, Morishita $\mathrm{R}$, Annex BH: Results of a double-blind, placebo-controlled study to assess the safety of intramuscular injection of hepatocyte growth factor plasmid to improve limb perfusion in patients with critical limb ischemia. Circulation 2008, 118:58-65.

20. Marston W, Powell R, Dall'Olmo C, Guzman R, Moore CJ, Martinez J, Halloran B, Henke P, Stiff P, Comerota A, Velazquez O, Tzeng E, Mendelsohn F, Henry T, Bohannon WT, Saucedo J, Bartel R, Longcore A, Stern T, Watling S: Patientspecific cellular therapy (Ixmyelocel-T) is safe and improves time to treatment failure in patients with critical limb ischemia and no revascularization options. Circulation 2011, 124:A8547.

21. Powell RJ, Marston W, Berceli SA, Guzman R, Henry T, Longcore A, Stern T, Watling S, Bartel R: Cellular therapy with ixmyelocel-T to treat critical limb ischemia: the randomized, double-blind, placebo-controlled RESTORE-CLI trial. Mol Ther 20:1280-1286

doi:10.1186/scrt117

Cite this article as: Bartel RL, et al:: The Aastrom experience. Stem Cell Research \& Therapy 2012, 3:26. 